\title{
Langevin equation involving one fractional order with three- point boundary conditions
}

\author{
Ahmed Salem ${ }^{a, *}$, Faris Alzahrani ${ }^{a}$, Lamya Almaghamsi ${ }^{a, b}$ \\ ${ }^{a}$ Department of Mathematics, Faculty of Science, King Abdulaziz University, P. O. Box 80203, Jeddah, 21589, Saudi Arabia. \\ ${ }^{b}$ Department of Mathematics, University of Jeddah, 41510, Saudi Arabia.
}

\begin{abstract}
In this paper, we investigate a class of nonlinear Langevin equation involving one fractional order $\alpha \in(0,1]$ with three-point boundary conditions. By the Banach contraction principle and Krasnoselskii's fixed point theorem, the existence and uniqueness results of solutions are obtained. Two examples are given to show the applicability of our main results.
\end{abstract}

Keywords: Fractional Langevin equations, fixed point theorem, existence and uniqueness.

2010 MSC: 26A33, 34A08, 34A12, 34B15.

(C)2019 All rights reserved.

\section{Introduction}

Fractional calculus has been extensively studied and developed during the last few decades due to its important application in many areas. It has become a new research field in differential equations $[9,16,23,26]$. The Langevin equation (first formulated by Langevin in 1908) is found to be an effective tool to describe the evolution of physical phenomena in fluctuating environments [6]. As the intensive development of fractional derivative, the fractional Langevin equations have been introduced by Mainardi and Pironi [14]. The general form of the nonlinear fractional Langevin equations is presented as

$$
{ }^{c} D^{\alpha}\left({ }^{c} D^{\beta}+\gamma\right) u(t)=f(t, u(t)),
$$

where ${ }^{c} D^{\alpha}$ and ${ }^{c} D^{\beta}$ are the Caputo fractional derivatives and $f:[0,1] \times \mathbb{R} \rightarrow \mathbb{R}$ is a given continuously differentiable function, $m-1<\alpha \leqslant m$ and $n-1<\beta \leqslant n, m, n \in \mathbb{N}$ [22]. It worth mentioning that mainly, fractional Langevin equations have been studied extensively. Recently, the existence and uniqueness solution for the nonlinear fractional Langevin equations involving two fractional orders was studied in $[1-8,11,12,15,17,21-25,27]$ and the extensive list of references given therein. With different unit intervals of values to two fractional orders $\alpha$ and $\beta$, many authors introduced their works. For instance, $[3,4,22,24,25]$ concerned with $m-1<\alpha \leqslant m$ and $n-1<\beta \leqslant n, m, n \in \mathbb{N},[1,15,21]$

\footnotetext{
${ }^{*}$ Corresponding author

Email address: ahmedsalem74@hotmail.com (Ahmed Salem)
}

doi: $10.22436 /$ jnsa.012.12.02

Received: 2019-04-02 Revised: 2019-05-31 Accepted: 2019-06-16 
concerned with $0<\alpha, \beta \leqslant 1,[2,7,12,27]$ concerned with $0<\alpha \leqslant 1$ and $1<\beta \leqslant 2,[5,11]$ concerned with $1<\alpha \leqslant 2$ and $0<\beta \leqslant 1$, and Gao et al [8] concerned with $1<\alpha, \beta \leqslant 2$.

Inspired by the papers mentioned above, in this paper we consider the nonlinear fractional Langevin equations

$$
{ }^{c} D^{\alpha}\left(D^{2}+\lambda^{2}\right) u(t)=f(t, u(t)), \quad t \in[0,1],
$$

subject to the three-point boundary conditions

$$
u(0)=0, \quad u^{\prime \prime}(0)=0, \quad u(1)=\beta u(\eta),
$$

where ${ }^{c} D^{\alpha}$ is the Caputo fractional derivative of fractional order $\alpha \in(0,1]$, $D^{2}$ is the second ordinary derivative, $f:[0,1] \times \mathbb{R} \rightarrow \mathbb{R}$ is a continuously differentiable function, and $\lambda \in \mathbb{R}_{+}$and $\beta \in \mathbb{R}$ such that:

$$
\beta \neq \frac{\sin \lambda}{\sin \lambda \eta} \text {. }
$$

Some of the most elegant examples of Chua's circuit and its many variants were motivated by the discovery of the simple third-order differential equations of the form $\dddot{x}=F(\ddot{x}, \dot{x}, x)$ whose solutions are chaotic $[18,20]$. The systems of the former form have been called Jerk equations (time derivative of acceleration) [19]. A simple Jerk circuit that allows studying chaotic dynamics in terms of theoretical variables $x, \dot{x}, \ddot{x}$ and $\ddot{x}$ and their equivalent experimental outputs was modeled as [13]

$$
\dddot{x}+A_{1} \ddot{x}+A_{2} \dot{x}+A_{3}|x|+A_{4}=0
$$

with $A_{i},(i=1,2,3,4)$ being control parameters might behave chaotically for suitably chosen control parameter values and initial conditions.

Our interest in studying the Langevin problem (1.1) comes from its application as a model for physical systems exhibiting anomalous diffusion. In fact, it is well known that in many cases the most convenient way of describing the time evolution of the velocity of the Brownian motion is to use the Langevin equation [7]. It is worth pointing out that the equation (1.1) can be considered as a fractional form of the Jerk chaotic equations mentioned above. Therefore, our work can be considered as a contribution of development class of chaotic electrical circuit.

In the beginning of the paper we provide some basic concepts on the fractional integrals and derivatives. Then, we use the Banach contraction principle and Krasnoselskii's fixed point theorem to study the existence and uniqueness solution of the three points boundary problem (1.1)-(1.2).

\section{Preliminaries}

Here, we recall several known definitions and properties from fractional calculus theory. For details, see $[9,16]$.

Definition 2.1. The Riemann-Liouville fractional integral of order $\alpha>0$ for a continuous function $f$ : $[0, \infty) \rightarrow \mathbb{R}$ is defined as

$$
I^{\alpha} f(t)=\int_{0}^{t} \frac{(t-s)^{\alpha-1}}{\Gamma(\alpha)} f(s) d s,
$$

where $\Gamma$ (.) denotes the Gamma function, provided that the right-hand-side integral exists.

Definition 2.2. Let $n \in \mathbb{N}$ be a positive integer and $\alpha$ be a positive real such that $n-1<\alpha \leqslant n$, then the fractional derivative of a function $f:[0, \infty) \rightarrow \mathbb{R}$ in the Caputo sense is defined as

$$
{ }^{c} D^{\alpha} f(t)=\frac{1}{\Gamma(n-\alpha)} \int_{0}^{t}(t-s)^{n-\alpha-1} f^{(n)}(s) d s,
$$

provided that the right-hand-side integral exists and is finite. We notice that the Caputo derivative of a constant is zero. 
Lemma 2.3. Let $\mathrm{n} \in \mathbb{N}$ and $\mathrm{n}-1<\alpha \leqslant n$. If $\mathrm{u}$ is a continuous function on $[0,1]$, then we have

$$
I^{\alpha c} D^{\alpha} u(t)=u(t)+c_{0}+c_{1} t+\cdots+c_{n-1} t^{n-1} .
$$

Lemma 2.4. The unique solution of the fractional differential equation

$$
{ }^{c} D^{\alpha}\left(D^{2}+\lambda^{2}\right) u(t)=\theta(t), \quad n-1<\alpha \leqslant n, n \in \mathbb{N},
$$

where $\theta$ is a continuous function on $[0,1]$, given by

$$
u(t)=\frac{1}{\lambda} \int_{0}^{t} \sin \lambda(t-s)\left(\int_{0}^{s} \frac{(s-\tau)^{\alpha-1}}{\Gamma(\alpha)} \theta(\tau) d \tau+\sum_{i=1}^{n-1} c_{i} s^{i}\right) d s+c_{n} \cos \lambda t+c_{n+1} \cos \lambda t,
$$

where $c_{i}, i=0,1, \ldots, n+1$ are constants.

Proof. Assume that $u(t)$ satisfies (2.1), then Lemma 2.3 and the variation of parameter method imply the desired results.

Lemma 2.5. The function $\mathrm{u}(\mathrm{t})$ is a unique solution of the boundary value problem (1.1)-(1.2) if and only if it is a solution of the nonlinear mixed Fredholm-Volterra integral equation

$$
\begin{aligned}
u(t)= & \frac{1}{\lambda} \int_{0}^{t} \sin \lambda(t-s)\left(\int_{0}^{s} \frac{(s-\tau)^{\alpha-1}}{\Gamma(\alpha)} f(\tau, u(\tau)) d \tau\right) d s \\
& +\frac{\sin \lambda t}{\Delta}\left[\beta \int_{0}^{\eta} \sin \lambda(\eta-s)\left(\int_{0}^{s} \frac{(s-\tau)^{\alpha-1}}{\Gamma(\alpha)} f(\tau, u(\tau)) d \tau\right) d s\right. \\
& \left.-\int_{0}^{1} \sin \lambda(1-s)\left(\int_{0}^{s} \frac{(s-\tau)^{\alpha-1}}{\Gamma(\alpha)} f(\tau, u(\tau)) d \tau\right) d s\right]
\end{aligned}
$$

where

$$
\Delta=\lambda(\sin \lambda-\beta \sin \lambda \eta) \neq 0 .
$$

Proof. Applying Lemma 2.4, when $0<\alpha \leqslant 1$, we get

$$
u(t)=c_{1} \cos \lambda t+c_{2} \sin \lambda t+\frac{c_{0}}{\lambda^{2}}(1-\cos \lambda t)+\frac{1}{\lambda} \int_{0}^{t} \sin \lambda(t-s)\left(\int_{0}^{s} \frac{(s-\tau)^{\alpha-1}}{\Gamma(\alpha)} f(\tau, u(\tau)) d \tau\right) d s .
$$

Using the boundary condition (1.2), we find that $c_{0}=c_{1}=0$, and

$$
c_{2}=\frac{1}{\Delta}\left[\int_{0}^{\eta} \sin \lambda(\eta-s)\left(\int_{0}^{s} \frac{(s-\tau)^{\alpha-1}}{\Gamma(\alpha)} \theta(\tau) d \tau\right) d s-\int_{0}^{1} \sin \lambda(1-s)\left(\int_{0}^{s} \frac{(s-\tau)^{\alpha-1}}{\Gamma(\alpha)} \theta(\tau) d \tau\right) d s\right]
$$

Substituting these values of $c_{0}, c_{1}, c_{2}$ in (2.4), we get (2.2). This completes the proof.

\section{Existence of solution}

Let $\mathbb{E}=C([0,1], \mathbb{R})$ be the Banach space of all continuous functions from $[0,1] \longrightarrow \mathbb{R}$ endowed the norm defined by

$$
\|u\|=\sup \{|u(t)|, t \in[0,1]\} .
$$

For the sake of convenience, we set

$$
B=\left|\frac{\Delta_{1}(\lambda+1)+\lambda \beta_{1} \eta^{\alpha+1}}{\lambda \Delta_{1} \Gamma(\alpha+2)}\right|
$$


where $\beta_{1}=|\beta|, \Delta_{1}=|\Delta|$ and $\Delta$ is given by (2.3). In view of Lemma 2.5, we transform problem (1.1)-(1.2) as

$$
u=T(u),
$$

where $\mathrm{T}: \mathbb{E} \longrightarrow \mathbb{E}$ is defined by

$$
\begin{aligned}
(\mathrm{Tu})(\mathrm{t})= & \frac{1}{\lambda} \int_{0}^{t} \sin \lambda(t-s)\left(\int_{0}^{s} \frac{(s-\tau)^{\alpha-1}}{\Gamma(\alpha)} f(\tau, u(\tau)) d \tau\right) d s \\
& +\frac{\sin \lambda t}{\Delta}\left[\beta \int_{0}^{\eta} \sin \lambda(\eta-s)\left(\int_{0}^{s} \frac{(s-\tau)^{\alpha-1}}{\Gamma(\alpha)} f(\tau, u(\tau)) d \tau\right) d s\right. \\
& \left.-\int_{0}^{1} \sin \lambda(1-s)\left(\int_{0}^{s} \frac{(s-\tau)^{\alpha-1}}{\Gamma(\alpha)} f(\tau, u(\tau)) d \tau\right) d s\right] .
\end{aligned}
$$

Observe that problem (1.1)-(1.2) has solutions if the operator (3.2) has fixed points.

Theorem 3.1. Let $f:[0,1] \times \mathbb{R} \longrightarrow \mathbb{R}$ be a jointly continuous function satisfying the condition

$$
\left|f\left(t, u_{1}\right)-f\left(t, u_{2}\right)\right| \leqslant K\left|u_{1}-u_{2}\right|, \quad \forall t \in[0,1], x, y \in \mathbb{R},
$$

where $K$ is the Lipschitz constant .Then the boundary value problem (1.1)-(1.2) has a unique solution if $B<1 / K$, where $\mathrm{B}$ is given by (3.1).

Proof. As a first step, for $T$ defined by (3.2), we show that $T \mathcal{B}_{r} \subset \mathcal{B}_{r}$, where $\mathcal{B}_{r}=\{u \in \mathbb{E}:\|u\| \leqslant r\}$. For that, set $\sup _{t \in[0,1]}|u(t, 0)|=\sigma$ and choose $r \geqslant(\sigma B) /(1-K B)$, where $B$ is given by (3.1). For $u \in \mathcal{B}_{r}$, we have

$$
\begin{aligned}
& \|(T u)(t)\|=\sup _{t \in[0,1]} \mid \frac{1}{\lambda} \int_{0}^{t} \sin \lambda(t-s)\left(\int_{0}^{s} \frac{(s-\tau)^{\alpha-1}}{\Gamma(\alpha)} f(\tau, u(\tau)) d \tau\right) d s \\
& +\frac{\sin \lambda \mathrm{t}}{\Delta}\left[\beta \int_{0}^{\eta} \sin \lambda(\eta-s)\left(\int_{0}^{s} \frac{(s-\tau)^{\alpha-1}}{\Gamma(\alpha)} f(\tau, u(\tau)) d \tau\right) d s\right. \\
& \left.-\int_{0}^{1} \sin \lambda(1-s)\left(\int_{0}^{s} \frac{(s-\tau)^{\alpha-1}}{\Gamma(\alpha)} f(\tau, u(\tau)) d \tau\right) d s\right] \mid \\
& \leqslant \sup _{t \in[0,1]}\left(\frac{1}{\lambda} \int_{0}^{t}\left(\int_{0}^{s} \frac{(s-\tau)^{\alpha-1}}{\Gamma(\alpha)}(|f(\tau, u(\tau))-f(\tau, 0)|+|f(\tau, 0)|) d \tau\right) d s\right) \\
& +\frac{1}{|\Delta|}\left[|\beta| \int_{0}^{\eta}\left(\int_{0}^{s} \frac{(s-\tau)^{\alpha-1}}{\Gamma(\alpha)}(|f(\tau, u(\tau))-f(\tau, 0)|+|f(\tau, 0)|) d \tau\right) d s\right. \\
& \left.+\int_{0}^{1}\left(\int_{0}^{s} \frac{(s-\tau)^{\alpha-1}}{\Gamma(\alpha)}(|\mathbf{f}(\tau, \mathbf{u}(\tau))-\mathbf{f}(\tau, 0)|+|\mathbf{f}(\tau, 0)|) d \tau\right) \mathrm{ds}\right] \\
& \leqslant \sup _{t \in[0,1]}\left(\frac{1}{\lambda} \int_{0}^{t}\left(\int_{0}^{s} \frac{(s-\tau)^{\alpha-1}}{\Gamma(\alpha)}(K|\mathfrak{u}(\tau)|+|f(\tau, 0)|) d \tau\right) d s\right) \\
& +\frac{1}{|\Delta|}\left[|\beta| \int_{0}^{\eta}\left(\int_{0}^{s} \frac{(s-\tau)^{\alpha-1}}{\Gamma(\alpha)}(K|u(\tau)|+|f(\tau, 0)|) d \tau\right) d s\right. \\
& \left.+\int_{0}^{1}\left(\int_{0}^{s} \frac{(s-\tau)^{\alpha-1}}{\Gamma(\alpha)}(K|u(\tau)|+|f(\tau, 0)|) d \tau\right) d s\right] \\
& \leqslant(K r+\sigma)\left(\frac{1}{\lambda} \sup _{t \in[0,1]} \int_{0}^{t}+\frac{|\beta|}{|\Delta|} \int_{0}^{\eta}+\int_{0}^{1}\right)\left(\int_{0}^{s} \frac{(s-\tau)^{\alpha-1}}{\Gamma(\alpha)} d \tau\right) d s \\
& \leqslant(K r+\sigma)\left|\frac{\Delta_{1}(\lambda+1)+\lambda \beta_{1} \eta^{\alpha+1}}{\lambda \Delta_{1} \Gamma(\alpha+2)}\right|=(K r+\sigma) B \leqslant r .
\end{aligned}
$$


Now, let $u_{1}, u_{2} \in \mathbb{E}$ for each $t \in[0,1]$, we obtain

$$
\begin{aligned}
\left\|\left(T u_{1}\right)(t)-\left(T u_{2}\right)(t)\right\|= & \sup _{t \in[0,1]}\left|\left(T u_{1}\right)(t)-\left(T u_{2}\right)(t)\right| \\
\leqslant & \sup _{t \in[0,1]}\left\{\left(\frac{1}{\lambda} \int_{0}^{t}\left(\int_{0}^{s} \frac{(s-\tau)^{\alpha-1}}{\Gamma(\alpha)}\left|f\left(\tau, u_{1}(\tau)\right)-f\left(\tau, u_{2}(\tau)\right)\right| d \tau\right) d s\right)\right. \\
& +\frac{1}{\Delta_{1}}\left[\beta_{1} \int_{0}^{\eta}\left(\int_{0}^{s} \frac{(s-\tau)^{\alpha-1}}{\Gamma(\alpha)}\left|f\left(\tau, u_{1}(\tau)\right)-f\left(\tau, u_{2}(\tau)\right)\right| d \tau\right) d s\right. \\
& \left.\left.+\int_{0}^{1}\left(\int_{0}^{s} \frac{(s-\tau)^{\alpha-1}}{\Gamma(\alpha)}\left|f\left(\tau, u_{1}(\tau)\right)-f\left(\tau, u_{2}(\tau)\right)\right| d \tau\right) d s\right]\right\} \\
\leqslant & K\left\|u_{1}-u_{2}\right\|\left(\frac{1}{\lambda} \sup _{t \in[0,1]} \int_{0}^{t}+\frac{|\beta|}{|\Delta|} \int_{0}^{\eta}+\int_{0}^{1}\right)\left(\int_{0}^{s} \frac{(s-\tau)^{\alpha-1}}{\Gamma(\alpha)} d \tau\right) d s \\
= & B K\left\|u_{1}-u_{2}\right\| .
\end{aligned}
$$

By virtue that $B<1 / K$, then the operator $T$ is a contraction. Therefore, the conclusion of the theorem follows by the contraction mapping principle. This ends the proof.

Now we study the existence of solutions for boundary value problem (1.1)-(1.2) by satisfying the conditions due to Krasnoselskii's fixed point theorem that is as follows.

Theorem 3.2 ([10]). Let $\Omega$ be a closed convex and nonempty subset of Banach space $\mathbb{E}$. Let $\mathcal{F}_{1}$ and $\mathcal{F}_{2}$ be two operators such that:

1. $\mathcal{F}_{1} z+\mathcal{F}_{2} z \in \Omega$;

2. $\mathcal{F}_{1}$ is compact and continuous;

3. $\mathcal{F}_{2}$ is a contraction mapping.

Then there exists $z \in \Omega$ such that $z=\mathcal{F}_{1} z+\mathcal{F}_{2} z$.

Theorem 3.3. Assume that $\mathrm{f}:[0,1] \times \mathbb{R} \longrightarrow \mathbb{R}$ be a jointly continuous function and the following assumptions hold:

$\left(\mathrm{H}_{1}\right)\left|\mathrm{f}\left(\mathrm{t}, \mathrm{u}_{1}\right)-\mathrm{f}\left(\mathrm{t}, \mathrm{u}_{2}\right)\right| \leqslant K\left|\mathrm{u}_{1}-\mathrm{u}_{2}\right|, \quad \forall \mathrm{t} \in[0,1], \mathrm{u}_{1}, \mathrm{u}_{2} \in \mathbb{R} ;$

$\left(\mathrm{H}_{2}\right)|\mathrm{f}(\mathrm{t}, \mathrm{u})| \leqslant \omega(\mathrm{t})$ for all $(\mathrm{t}, \mathrm{u}) \in[0,1] \times \mathbb{R}$ with $\omega \in \mathrm{C}[0,1]$.

Then the boundary value problem (1.1)-(1.2) has at least one solution in $[0,1]$ if $\Lambda<1 / K$, where $\Lambda$ is given by

$$
\Lambda=\frac{\beta_{1} \eta^{\alpha+1}+\Delta_{1}}{\Delta_{1} \Gamma(\alpha+2)}
$$

$\beta_{1}=|\beta|$, and $\Delta_{1}=|\Delta|$.

Proof. Set $\sup _{t \in[0,1]}|\omega(t)| \leqslant\|\omega\|$ and consider $\mathcal{B}_{r}=\{u \in \mathbb{E}:\|u\| \leqslant r\}$ with fix

$$
r \geqslant\left|\frac{\Delta_{1}(\lambda+1)+\lambda \beta_{1} \eta^{\alpha+1}}{\lambda \Delta_{1} \Gamma(\alpha+2)}\right|\|\omega\|
$$

Define the two operators $T_{1}$ and $T_{2}$ on $\mathcal{B}_{r}$ as

$$
\begin{aligned}
& \left(T_{1} u\right)(t)=\frac{1}{\lambda} \int_{0}^{t} \sin \lambda(t-s)\left(\int_{0}^{s} \frac{(s-\tau)^{\alpha-1}}{\Gamma(\alpha)} f(\tau, u(\tau)) d \tau\right) d s \\
& \left(T_{2} u\right)(t)=\frac{\sin \lambda t}{\Delta}\left[\beta \int_{0}^{\eta} \sin \lambda(\eta-s)\left(\int_{0}^{s} \frac{(s-\tau)^{\alpha-1}}{\Gamma(\alpha)} f(\tau, u(\tau)) d \tau\right) d s\right.
\end{aligned}
$$




$$
\left.-\int_{0}^{1} \sin \lambda(1-s)\left(\int_{0}^{s} \frac{(s-\tau)^{\alpha-1}}{\Gamma(\alpha)} f(\tau, u(\tau)) d \tau\right) d s\right] .
$$

For $\mathfrak{u}_{1}, \mathrm{u}_{2} \in \mathcal{B}_{\mathrm{r}}$, it follows from (3.2) that

$$
\left\|\mathrm{T}_{1} \mathrm{u}_{1}+\mathrm{T}_{2} \mathrm{u}_{2}\right\| \leqslant\left|\frac{\Delta_{1}(\lambda+1)+\lambda \beta_{1} \eta^{\alpha+1}}{\lambda \Delta_{1} \Gamma(\alpha+2)}\right|\|\omega\| \leqslant \mathrm{r} .
$$

Therefore, $T_{1} u_{1}+T_{2} u_{2} \in \mathcal{B}_{r}$. By virtue of $\Lambda<1 / K$ it can be easily shown that $T_{2}$ is a contraction mapping. The continuity of the operator $T_{1}$ comes from the continuity of the function $f$. Also, $T_{1}$ is uniformly bounded on $\mathcal{B}_{r}$ as

$$
\left\|T_{1} u\right\| \leqslant \frac{\|\omega\|}{\lambda \Gamma(\alpha+2)} .
$$

To prove the compactness of the operator $T_{1}$, assume that $D=[0,1] \times \mathcal{B}_{r} \subset \mathbb{E}$ and define $\sup _{(t, u) \in D}|f(t, u)|=$ $\mathrm{H}_{\mathrm{r}}$. Let

$$
\delta=\min \left\{\left[\frac{\epsilon \lambda \Gamma(\alpha+2)}{\mathrm{H}_{\mathrm{r}}(\lambda+\alpha+1)}\right]^{\frac{1}{\alpha}},\left[\frac{\epsilon \lambda \Gamma(\alpha+2)}{\mathrm{H}_{\mathrm{r}}\left(\lambda+2^{\alpha+1}\right)}\right]^{\frac{1}{\alpha+1}}\right\}, \forall \epsilon>0 .
$$

Thus, for all $u \in \mathcal{B}_{r}$ and $t_{1}, t_{2} \in[0,1]$ with $t_{1}<t_{2}$ and $t_{2}-t_{1}<\delta$

$$
\begin{aligned}
\left\|\left(T_{1} u\right)\left(t_{2}\right)-\left(T_{1} u\right)\left(t_{1}\right)\right\|= & \| \frac{1}{\lambda} \int_{0}^{t_{2}} \sin \lambda\left(t_{2}-s\right)\left(\int_{0}^{s} \frac{(s-\tau)^{\alpha-1}}{\Gamma(\alpha)} f(\tau, u(\tau)) d \tau\right) d s \\
& -\frac{1}{\lambda} \int_{0}^{t_{1}} \sin \lambda\left(t_{1}-s\right)\left(\int_{0}^{s} \frac{(s-\tau)^{\alpha-1}}{\Gamma(\alpha)} f(\tau, u(\tau)) d \tau\right) d s \| \\
\leqslant & \frac{H_{r}}{\lambda} \int_{0}^{t_{1}}\left|\sin \lambda\left(t_{2}-s\right)-\sin \lambda\left(t_{1}-s\right)\right|\left(\int_{0}^{s} \frac{(s-\tau)^{\alpha-1}}{\Gamma(\alpha)} d \tau\right) d s \\
& +\frac{H_{r}}{\lambda} \int_{t_{1}}^{t_{2}}\left(\int_{0}^{s} \frac{(s-\tau)^{\alpha-1}}{\Gamma(\alpha)} d \tau\right) d s \\
= & \frac{H_{r}}{\lambda \Gamma(\alpha+1)}\left[\lambda \int_{0}^{t_{1}} s^{\alpha}\left|\int_{t_{1}}^{t_{2}} \cos \lambda(\xi-s) d \xi\right| d s+\int_{t_{1}}^{t_{2}} s^{\alpha} d s\right] \\
\leqslant & \frac{H_{r}}{\lambda \Gamma(\alpha+2)}\left[\lambda\left(t_{2}-t_{1}\right) t_{1}^{\alpha+1}+t_{2}^{\alpha+1}-t_{1}^{\alpha+1}\right],
\end{aligned}
$$

which is independent of $u$ and tends to zero when $t_{2} \longrightarrow t_{1}$. It obvious that $t_{2}>\delta$ and there are two probabilities for $t_{1}$ and $\delta$.

Case I: $\delta \leqslant t_{1}<t_{2}<1$, the mean value theorem implies that there exists $t \in\left(t_{1}, t_{2}\right)$ such that

$$
t_{2}^{\alpha+1}-t_{1}^{\alpha+1}=(\alpha+1)\left(t_{2}-t_{1}\right) t^{\alpha}<(\alpha+1) \delta t^{\alpha-1} t<(\alpha+1) \delta^{\alpha} .
$$

Hence, we obtain

$$
\left\|\left(T_{1} u\right)\left(t_{2}\right)-\left(T_{1} u\right)\left(t_{1}\right)\right\| \leqslant \frac{H_{r}}{\lambda \Gamma(\alpha+2)}\left(\lambda \delta t_{1}^{\alpha-1} t_{1}^{2}+(\alpha+1) \delta^{\alpha}\right)<\frac{H_{r}(\lambda+\alpha+1)}{\lambda \Gamma(\alpha+2)} \delta^{\alpha}<\epsilon .
$$

Case II: $0 \leqslant t_{1}<\delta<t_{2}<1$ and so $t_{2}<2 \delta$. These imply that

$$
\left\|\left(T_{1} u\right) t_{2}-\left(T_{1} u\right) t_{1}\right\| \leqslant \frac{H_{r}}{\lambda \Gamma(\alpha+2)}\left(\lambda \delta^{\alpha+2}+(2 \delta)^{\alpha+1}\right)<\frac{H_{r}\left(\lambda+2^{\alpha+1}\right)}{\lambda \Gamma(\alpha+2)} \delta^{\alpha+1}<\epsilon .
$$

Therefore, the operator $T_{1}$ is relatively compact on $\mathcal{B}_{r}$. Hence by the Arzela-Ascoli Theorem, the operator $\mathrm{T}_{1}$ is compact on $\mathcal{B}_{\mathrm{r}}$. Thus all assumptions of Theorem 3.3 are satisfied and the conclusion of Theorem 3.2 implies that the boundary value problem (1.1) and (1.2) has at least one solution in $[0,1]$. 


\section{Examples}

We present two examples to better illustrate our main results.

Example 4.1. Consider the following initial value problem

$$
\left\{\begin{array}{l}
{ }^{c} D^{\frac{1}{2}}\left(D^{2}+16\right) u(t)=k\left(t^{\frac{1}{2}}-\sin (t)+\tan ^{-1} u(t)\right), \quad 0<t<1,0<\alpha \leqslant 1, \\
u(0)=0, \quad u^{\prime \prime}(0)=0, \quad u(1)=2 u\left(\frac{1}{2}\right) .
\end{array}\right.
$$

Here, $f(t, u(t))=K\left(t^{\frac{1}{2}}-\sin (t)+\tan ^{-1} u(t)\right), \lambda=4, \beta=2, \eta=\frac{1}{2}$. Clearly

$$
\left|f\left(t, u_{1}(t)\right)-f\left(t, u_{2}(t)\right)\right| \leqslant K\left|u_{1}-u_{2}\right|
$$

and

$$
\mathrm{B}=\frac{5 \Delta_{1}+2\left(\frac{1}{2}\right)^{\left(\frac{5}{2}\right)}}{3 \sqrt{\pi} \Delta_{1}} .
$$

For $\mathrm{K}<1.056222342$, it follows by Theorem 3.1 that problem (4.1) has a unique solution.

Example 4.2. Consider the following initial value problem

$$
\left\{\begin{array}{l}
{ }^{c} D^{\frac{3}{4}}\left(D^{2}+9\right) u(t)=K(\cos (t) u(t)-1), \quad 0<t<1,0<\alpha \leqslant 1, \\
u(0)=0, \quad u^{\prime \prime}(0)=0, \quad u(1)=3 u\left(\frac{2}{5}\right) .
\end{array}\right.
$$

Here, $f(t, u(t))=K(\cos (t) u(t)-1), \lambda=3, \beta=3, \eta=\frac{2}{5}$. Clearly

$$
\left|f\left(t, u_{1}(t)\right)-f\left(t, u_{2}(t)\right)\right| \leqslant K\left|u_{1}-u_{2}\right|
$$

and

$$
\mathrm{B}=\frac{4 \Delta_{1}+3\left(\frac{2}{5}\right)^{\left(\frac{11}{4}\right)}}{3 \Delta_{1} \Gamma\left(2+\frac{3}{4}\right)} .
$$

For $\mathrm{K}<1.197197490$, it follows by Theorem 3.1 that problem (4.2) has a unique solution.

\section{Conclusion}

The existence and uniqueness of solutions for three-point boundary value problem involving Langevin equation with one fractional orders has been discussed. We apply the concepts of fractional calculus together with fixed point theorems to establish the existence and uniqueness results. To investigate our problem, we apply Banach contraction principle and Krasnoselskii's fixed point theorem. Our approach is simple and is applicable to a variety of real world problems.

As a special case, the existence results for a nonlinear third-order differential equations with a threepoint nonlinear boundary value problem

$$
\left(D^{3}+\lambda^{2} D\right) u(t)=f(t, u(t)), \quad t \in[0,1],
$$

subject to the three-point boundary conditions

$$
u(0)=0, \quad u^{\prime \prime}(0)=0, \quad u(1)=\beta u(\eta),
$$

can be obtained by fixing $\alpha=1$ in the results of this paper which can be considered as a special case of a simple Jerk Chaotic circuit equation (1.3). 


\section{Acknowledgment}

This article was supported by the Deanship of Scientific Research (DSR), King Abdulaziz University, Jeddah. The authors, therefore, acknowledge with thanks DSR technical and financial support.

\section{References}

[1] B. Ahmad, J. J. Nieto, Solvability of Nonlinear Langevin Equation Involving Two Fractional Orders with Dirichlet Boundary Conditions, Int. J. Differ. Equ., 2010 (2010), 10 pages. 1

[2] B. Ahmad, J. J. Nieto, A. Alsaedi, M. El-Shahed, A study of nonlinear Langevin equation involving two fractional orders in different intervals, Nonlinear Anal. Real World Appl., 13 (2012), 599-606. 1

[3] O. Baghani, On fractional Langevin equation involving two fractional orders, Commun. Nonlinear Sci. Numer. Simul., 42 (2017), 675-681. 1

[4] H. Baghani, Existence and uniqueness of solutions to fractional Langevin equations involving two fractional orders, J. Fixed Point Theory Appl., 20 (2018), 7 pages. 1

[5] A. Chen, Y. Chen, Existence of Solutions to Nonlinear Langevin Equation Involving Two Fractional Orders with Boundary Value Conditions, Bound. Value Probl., 2011 (2011), 17 pages. 1

[6] W. T. Coffey, Y. P. Kalmykov, J. T. Waldron, The Langevin Equation: With Applications to Stochastic Problems in Physics, Chemistry and Electrical Engineering, World Scientific Publishing Co., River Edge, (2004). 1

[7] H. Fazli, J. J. Nieto, Fractional Langevin equation with anti-periodic boundary conditions, Chaos Solitons Fractals, 114 (2018), 332-337. 1, 1

[8] Z. Y. Gao, X. L. Yu, J. R. Wang, Nonlocal problems for Langevin-type differential equations with two fractional-order derivatives, Bound. Value Probl., 2016 (2016), 21 pages. 1

[9] A. A. Kilbas, H. M. Srivastava, J. J. Trujillo, Theory and applications of fractional differential equations, Elsevier Science B.V., Amsterdam, (2006). 1, 2

[10] M. A. Krasnoselskii, Two remarks on the method of successive approximations, Uspekhi Mat. Nauk (N.S.), 10 (1955), 123-127. 3.2

[11] X. Z. Li, M. Medved', J. R. Wang, Generalized Boundary Value Problems for Nonlinear Fractional Langevin Equations, Acta Univ. Palack. Olomuc. Fac. Rerum Natur. Math., 53 (2014), 85-100. 1

[12] B. X. Li, S. R. Sun, Y. Sun, Existence of solutions for fractional Langevin equation with infinite-point boundary conditions, J. Appl. Math. Comput., 53 (2017), 683-692. 1

[13] S. J. Linz, J. C. Sprott, Elementary chaotic flow, Phys. Lett. A, 259 (1999), 240-245. 1

[14] F. Mainradi, P. Pironi, The fractional Langevin equation: Brownian motion revisited, Extracta Math., 11 (1996), $140-154$. 1

[15] T. Muensawat, S. K. Ntouyas, J. Tariboon, Systems of generalized Sturm-Liouville and Langevin fractional differential equations, Adv. Difference Equ., 2017 (2017), 15 pages. 1

[16] I. Podlubny, Fractional Differential Equations: An introduction to fractional derivatives, fractional differential equations, to methods of their solution and some of their applications, Academic Press, San Diego, (1999). 1, 2

[17] A. Salem, F. Alzahrani, L. Almaghamsi, Fractional Langevin equation with nonlocal integral boundary condition, Mathematics, 7 (2019), 1-10. 1

[18] J. C. Sprott, Some simple chaotic jerk functions, Amer. J. Phys., 65 (1997), 537-543. 1

[19] J. C. Sprott, A new class of chaotic circuit, Phys. Lett. A, 266 (2000), 19-23. 1

[20] J. C. Sprott, A new chaotic Jerk circuit, IEEE Tran. Citcuit Syst. A, 58 (2011), 240-243. 1

[21] W. Sudsutad, J. Tariboon, Nonlinear fractional integro-differential Langevin equation involving two fractional orders with three-point multi-term fractional integral boundary conditions, J. Appl. Math. Comput., 43 (2013), 507-522. 1

[22] T. Yu, K. Deng, M. Luo, Existence and uniqueness of solutions of initial value problems for nonlinear Langevin equation involving two fractional orders, Commun. Nonlinear Sci. Numer. Simul., 19 (2014), 1661-1668. 1

[23] W. Yukunthorn, S. K. Ntouyas, J. Tariboon, Nonlinear fractional Caputo-Langevin equation with nonlocal RiemannLiouville fractional integral conditions, Adv. Difference Equ., 2014 (2014), 18 pages. 1

[24] C. B. Zhai, P. P. Li, Nonnegative Solutions of Initial Value Problems for Langevin Equations Involving Two Fractional Orders, Mediterr. J. Math., 15 (2018), 11 pages. 1

[25] C. B. Zhai, P. P. Li, H. Y. Li, Single upper-solution or lower-solution method for Langevin equations with two fractional orders, Adv. Difference Equ., 2018 (2018), 10 pages. 1

[26] K. H. Zhao, P. Gong, Existence of positive solutions for a class of higher-order Caputo fractional differential equation, Qual. Theory Dyn. Syst., 14 (2015), 157-171. 1

[27] Z. F. Zhou, Y. Qiao, Solutions for a class of fractional Langevin equations with integral and anti-periodic boundary conditions, Bound. Value Probl., 2018 (2018), 10 pages. 1 